\title{
Use of a saline gradient for the diagnosis of schistosomiasis
}

\author{
Paulo Marcos Z Coelho ${ }^{1 /+}$, Arnon D Jurberg ${ }^{2}$, Áureo A Oliveira ${ }^{1}$, Naftale Katz ${ }^{1}$ \\ 'Laboratório de Esquistossomose, Centro de Pesquisas René Rachou-Fiocruz, Av. Augusto de Lima 1715, 30190-002 Belo Horizonte, MG, \\ Brasil ${ }^{2}$ Laboratório de Patologia, Instituto Oswaldo Cruz-Fiocruz, Rio de Janeiro, RJ, Brasil
}

The development of novel methods for parasitological diagnosis that are both highly sensitive and low in cost has been strongly recommended by the World Health Organization. In this study, a new technique for diagnosis of schistosomiasis mansoni is proposed based on the differential sedimentation of eggs when subjected to a slow continuous flux of $3 \%$ saline solution through a porous plaque. This influx suspends low-density faecal material, effectively cleaning the sample. The remaining sediment covering the porous plaque surface is then transferred to a glass slide and examined under a bright field microscope. Twelve Kato-Katz slides were used for comparison in the present study. Our results suggest that the saline gradient method detects a significantly higher number of eggs than the 12 Kato-Katz slides $(p<0.0001)$. We also found microscopic inspection to be quicker and easier with our newly described method. After cleaning the sample, the obtained sediment can also be conserved in a 10\% formaldehyde solution and examined for at least 45 days later without statistically significant egg count differences.

Key words: Schistosoma mansoni - diagnosis - faeces

Faecal exams are widely used for the diagnosis of intestinal helminth infections because microscopic findings of parasitic structures typically assure reliable and accurate identification of the parasites. Although several types of qualitative and quantitative methods exist for stool examination (Lutz 1919, Hastings-Willis 1921, Faust \& Melency 1924, Hoffman et al. 1934, Stoll 1946, Ritchie 1948, Sapero et al. 1951, Kato \& Miura 1954, Blagg et al. 1955, Bell 1963, Katz et al. 1972), some of these techniques have operational limitations, including high cost, complexity, low sensitivity and/or reproducibility. These limitations may restrict their respective uses in large-scale surveys in the field.

A method commonly used in epidemiological studies due to its relative simplicity and low cost is the quantitative Kato-Katz faecal smear technique (Katz et al. 1972). Katz et al. (1970) showed the limit of sensitivity for this method to be 20 eggs per gram of faeces by recovering Schistosoma mansoni eggs that were added to negative human faecal samples. This exam is considered to be the gold standard for diagnosing schistosomiasis mansoni as per the World Health Organization (WHO 1993). Although this method is widely used, the Kato-Katz test might lack sensitivity in situations of low worm burden, low endemicity and in cases of evaluation post treatment (Ferrari et al. 2003). To overcome these limitations, it is

Financial support: $\mathrm{CNPq}(\mathrm{PMZC}, \mathrm{ADJ}, \mathrm{AAO})$

The present device was patented by GESTEC/FIOCRUZ.

+ Corresponding author: coelhopm@cpqrr.fiocruz.br

Received 9 January 2009

Accepted 4 June 2009 necessary to increase the number of stool samples, which may increase costs and possibly cause logistic difficulties in epidemiological surveys (Enk et al. 2008).

Alternative approaches based on antibody, antigen and DNA detection are of potential application, but when the financial and infrastructural difficulties of most endemic countries are taken into consideration, these techniques become unsatisfactory (WHO 2006b). Perhaps as an opposite effect of successful control programs worldwide, the diagnosis of schistosomiasis still needs a more sensitive and low-cost tool for detecting light infections in the field (WHO 2006a). Recently, two novel methods have been proposed. Teixeira et al. (2007) developed a highly sensitive method (named Helmintex) for identifying $S$. mansoni eggs in large amounts of faeces. Faecal samples of $30 \mathrm{~g}$ were processed through a sequence of spontaneous sedimentation, sieving and the Ritchie method and then incubated with or without lectins. This was followed by isolation through interaction with paramagnetic beads and microscopic inspection. The other method consists of a hatching device containing a collecting container for the detection of miracidia (Jurberg et al. 2008). Although hatching methods are highly sensitive, they have an obvious limitation because the faecal samples must be fresh at the moment of the exam. On the other hand, the miracidia suspension fixed in $10 \%$ formaldehyde solution could be examined for at least 15 days after the fixation without a statistically significant loss of the miracidia number.

In an attempt to develop more sensitive diagnostic tools for schistosomiasis detection in the field, a simple saline gradient device was manufactured to facilitate egg detection by microscopic inspection. The present study evaluates the sensitivity of this new saline gradient device under experimental conditions, comparing it with the gold standard Kato-Katz method. This method was devised to be inexpensive, very sensitive, rapid and easy to perform. An additional positive aspect is a decrease in the total length of microscopic analysis. 


\section{MATERIALS AND METHODS}

The device - The saline gradient apparatus primarily consists of two interconnected cylindrical columns of transparent plastic. The columns were clawed at different heights for support. The reservoir column has a capacity for $50 \mathrm{~mL}$ of liquid and is placed higher. The separating column has the capacity for $10 \mathrm{~mL}$ of liquid and its superior aperture is the same height as the inferior end of the reservoir. A rubber hose with a roller clamp connects the columns. The bottom of the separating column has a tightly placed $1-\mathrm{cm}$-thick aquarium aerating stone sealed with silicon. In the upper part of the separating column there is a draining hose. A schematic drawing of the apparatus is presented in Fig. 1. In order to carry out a higher number of examinations at the same time, a modification for this device was also manufactured. The modification contains a large recipient reservoir and the $3 \%$ saline solution is placed in the centre of a circle defined by the 12 separating columns (Fig. 2). This arrangement was named the "spider" device.

The procedure - The device is set up as follows: (i) pre-wet the separating column with 3\% saline solution from the reservoir column by opening the roller clamp; (ii) stop the influx of the $3 \%$ saline solution to the separating column by closing the roller clamp; (iii) discard the excess saline just above the porous stone in the separation area using a pipette; (iv) with the use of a pipette, fill the separating column with a faecal suspension previously prepared by dilution of a $500 \mathrm{mg}$ stool sample in $3 \mathrm{~mL}$ of $0.9 \%$ saline solution and (v) open the roller clamp again, adjusting the saline flow to $10 \mathrm{drops} / \mathrm{min}$. The slow and continuous flow of the $3 \%$ saline solution from the reservoir column to the separating column causes a suspension and the discharge of low-density sewages, cleaning the sediment in the bottom of the latter column. Because the eggs have a higher density, they remain over the surface of the porous plate. The final sediment is further transferred to a glass slide and examined under a bright field microscope for the presence of eggs.

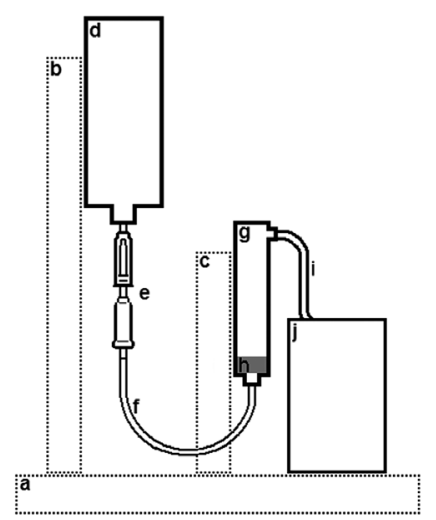

Fig. 1: the saline gradient method. Schematic drawing of a single saline gradient device in lateral view: a: base; b: bigger support arm; c: smaller support arm; d: saline reservoir; e: roller clamp; f: rubber hose; g: separating column; h: aerating stone (gray); i: draining hose; $\mathrm{j}$ : debris' collecting container.

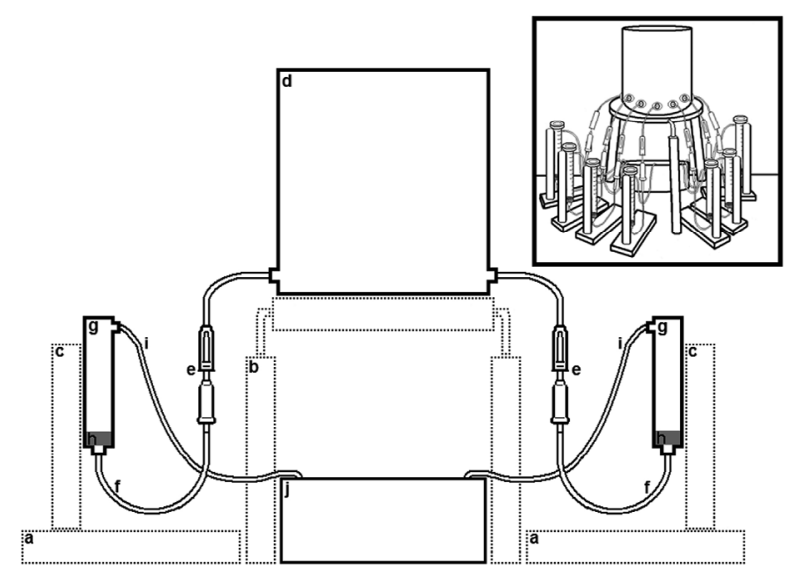

Fig. 2: the saline gradient method. Schematic drawing of the saline gradient "spider" arrangement in lateral view. Only two of 12 separating columns (g) are shown. a: base; b: saline support; c: support arm; d: saline reservoir; e: roller clamp; f: rubber hose; g: separating column; h: aerating stone (gray); i: draining hose; j: debris collecting container. Each separating column (g) and its matching base/support arm are arranged around the saline support, in a circular organization. The debris collecting container is placed soon below of the saline support, which stands the saline reservoir. Inset: schematic drawing of the saline-gradient "spider" arrangement.

Source of S. mansoni eggs - Fresh faeces from mice exposed to 50 cercariae of $S$. mansoni (LE strain) were used as an experimental source of eggs. The mice were all at least 50 days post-infection. A faecal suspension was produced in $10 \mathrm{~mL}$ of $0.9 \%$ cold saline solution using a glass rod in a plastic recipient. Five drops of $10 \%$ formaldehyde were also added to the recipient area in order to avoid miracidial hatching. The homogenised material was filtered with surgical gauze and folded into four layers into a sedimentation flask. After sedimentation of the material, the supernatant was aspirated by a pipette and discarded. The number of eggs within the sediment was not determined.

Addition of S. mansoni eggs to human faeces - Human faeces negative for $S$. mansoni were sieved through a $200 \mu \mathrm{m}$ stainless steel mesh (Arcelor - Mittal, Brazil). The total sediment from the mouse faeces was then mixed with $3 \mathrm{~g}$ of the sieved human faeces. This faecal mixture was further used for comparing both methods.

Comparison between the saline gradient and the Kato-Katz methods - To test the performance of the new saline gradient method, four samples of $500 \mathrm{mg}$ each were obtained from the faecal mixture mentioned above using a perforated steel plate. After setting the device (see "The procedure"), each diluted sample was gently applied with a Pasteur pipette into a separating column. After placement, the $3 \%$ saline influx was regulated to 10 drops/min by adjusting the roller clamp. The flowthrough reached the draining hose after approximately $20 \mathrm{~min}$ and the low-density debris began to pour into the waste container. When the cloudy solution had become more transparent and the sediment had been well defined (about $20 \mathrm{~min}$ ), the roller clamp was closed and 
the supernatant was carefully removed by aspirating it with a pipette. The sediment, in approximately $1-2 \mathrm{~mL}$ of saline, on the surface of the porous plate in each separating column was divided $(200 \mu \mathrm{L})$ and mounted between 5-10 regular flat glass slides and cover slips. Twelve Kato-Katz thick smears were then prepared (Katz et al. 1972) for comparison. The total amount of faeces in 12 slides corresponded to approximately $504 \mathrm{mg}$ (12 slides x $42 \mathrm{mg}$ ). Eggs were counted under a bright field microscope. The parasite burden was estimated using the ratio of eggs per gram of faeces, followed by multiplying the total number of eggs by two.

Statistical analysis - Considering that all faecal samples should have been positive for $S$. mansoni, the sensitivity for both methods was calculated by the number of positive findings divided by the total number of experiments $\left(\mathrm{n}_{1}=10\right)$. The arithmetical mean number of eggs per half gram of faeces in the saline gradient method and the total number of eggs per half gram of faeces in the Kato-Katz exam were the final result. The methods were compared using the paired T-test. To further evaluate whether the obtained sediment could be fixed for 45 days prior to the microscopic survey, fresh samples were compared to their matching fixed ones by using the Wilcoxon Signed Rank test $\left(\mathrm{n}_{2}=30\right.$ experiments). In both statistical tests, the significance level threshold was set to $\mathrm{p}<0.05$.

\section{RESULTS}

In both methods, all faecal samples $\left(\mathrm{n}_{1}=10\right)$ were positive for schistosomiasis ( $100 \%$ sensitivity) under the experimental conditions described above. The mean number of eggs detected in the saline gradient method was $6.83 \pm 1.41$ while the mean number of eggs found in 12 Kato-Katz thick smears was $4.70 \pm 1.25$. Thus, the saline gradient method detected a significantly higher number of eggs than the 12 Kato-Katz slides ( $\mathrm{p}<0.0001$, paired T-test) (Fig. 3). In addition, the time required for the inspection of the entire sediment (originally $500 \mathrm{mg}$ of faeces) obtained after the saline gradient procedure was about $20 \mathrm{~min}$, which corresponds to the time demanded for the examination of only four Kato-Katz slides.

In the comparison between fresh unfixed suspensions and their matching fixed ones that were analysed 45 days later, the mean number of eggs visualised was $7.22 \pm 1.35$ in the fresh samples and $7.05 \pm 2.30$ in the fixed samples. This difference was not statistically significant $(\mathrm{p}=$ 0.428, Wilcoxon Signed Rank test) (Fig. 4).

\section{DISCUSSION}

Saline solutions are widely used to suppress schistosome egg hatching. Moreover, these solutions have been previously noted to create a differential sedimentation gradient amongst $S$. mansoni eggs and tissue or faecal homogenates (Rowan \& Gram 1959, Ritchie \& BerriosDuran 1961). Schistosome eggs remain at the bottom of the flask, whilst low-density debris is suspended to the top of the gradient column. Our saline gradient apparatus was based on this principle and it is a simplification of the Ritchie and Berrios-Duran equipment for the mass recovery of schistosome eggs from tissues (Ritchie \&

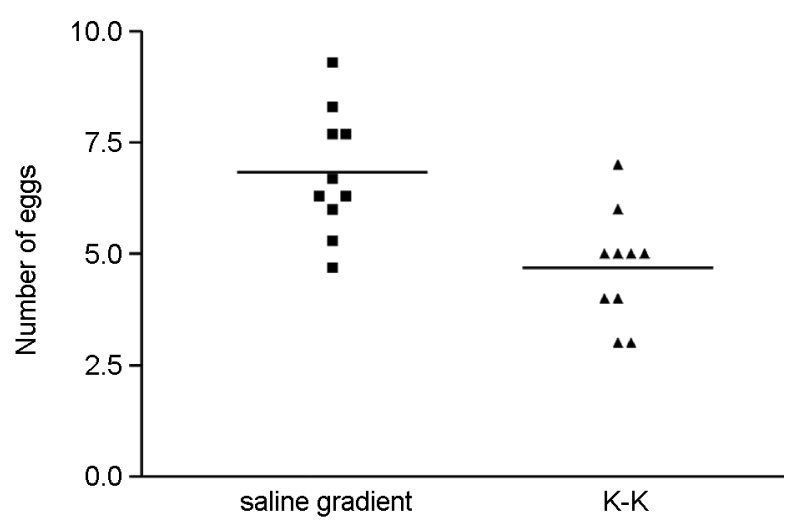

Fig. 3: number of Schistosoma mansoni eggs recovered in the saline gradient and in the Kato-Katz (K-K) methods under experimental conditions. Mean values were represented by a horizontal line. The number of eggs recovered by the saline gradient method was statistically higher than the number of eggs recovered by the analysis of 12 Kato-Katz slides per each comparison $(\mathrm{p}<0.0001)$.

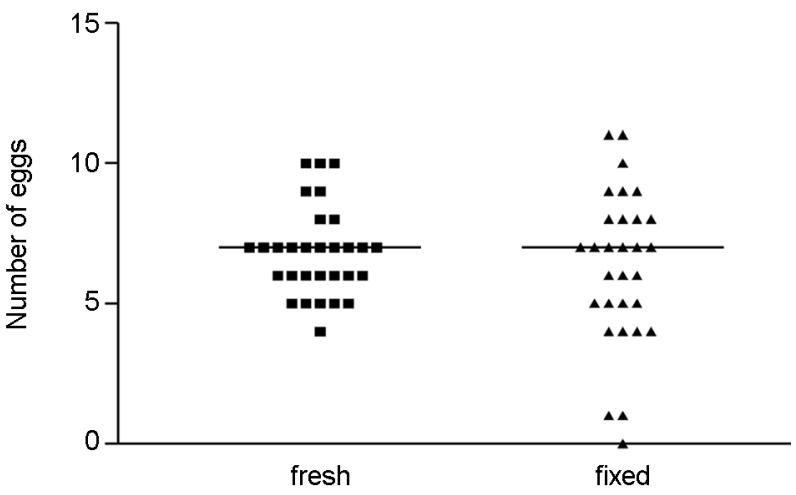

Fig. 4: number of Schistosoma mansoni eggs recovered by the saline gradient method in fresh and fixed samples under experimental conditions. Fecal samples were fixed in $10 \%$ formol (1:4) after had been cleaned in the saline gradient method and egg counting was performed 45 days later. Mean values were represented by a horizontal line. No significant statistical difference was observed.

Berrios-Duran 1961). Using this method, cleansed sediment remains on the surface of the thick aquarium aerating stone. This sediment is transferred to glass slides and the eggs can be easily and rapidly identified under bright field microscopy. It generally requires $20 \mathrm{~min}$ for the microscopic analysis of the entire sediment cleansed by the saline gradient, while only four Kato-Katz slides are usually examined within this period.

The present results, under laboratory conditions, show that the saline gradient method has a significantly higher recovery of eggs per gram of faeces than 12 Kato-Katz slides $(p<0.0001)$. This finding encouraged a preliminary field evaluation of the saline gradient technique in a small sample of 20 individuals (PMZ Coelho et al., unpublished observations). The selected group lives in the endemic area of Virgem das Graças, 
state of Minas Gerais (MG), Brazil. After several stool examinations by the Kato-Katz method and negative findings over years of studies, these patients had been referred to as "normal endemic" (Corrêa-Oliveira et al. 1998, Gazzinelli et al. 2006). The saline gradient method identified a positive infection rate for $S$. mansoni of $25 \%$ based on analysis of two faecal samples of $500 \mathrm{mg}$. On the other hand, two sets of 12 Kato-Katz slides per each individual revealed a prevalence of $20 \%$ of infection. Independent of the method performed, the amount of eggs per gram of faeces was very low in each infected patient (PMZ Coelho et al., unpublished observations), thus justifying the previous negative results and reinforcing the good performance of the new method.

Using the "spider" arrangement, another preliminary field survey was performed in Córrego Fundo, a region belonging to Comercinho, a municipality in MG. Fourteen positive findings were obtained after microscopic inspection of the obtained sediments collected from 40 individuals. The faecal sediments were kept in $10 \%$ formaldehyde for 60 days prior to the examination. Analysis of 12 Kato-Katz slides for each patient revealed 13 positive cases (PMZ Coelho et al., unpublished observations). The practicability and feasibility of the "spider" prototype allows an important improvement in field surveys, since 12 samples can be processed in parallel.

Taken together, these data stress the good performance of the new saline gradient technique for the diagnosis of $S$. mansoni. The experimental results presented here and the preliminary findings under field conditions suggest that the saline gradient method is more sensitive than 12 KatoKatz slides and its microscopic inspection is also quicker and easier. In addition, the saline gradient procedure only requires water and commercial sodium chloride and the device is reusable as many times as necessary as long as it is carefully washed. However, the use of a disposable separating column is under study and would likely facilitate large epidemiological surveys. Although the new saline gradient method appears to fit the recommendations of the last WHO report for novel diagnostic tools for schistosomiasis (WHO 2006a), additional field surveys are in progress to validate its use in large populations.

\section{ACKNOWLEDGEMENTS}

To Anna Carolina Lima, from Centro de Pesquisas René Rachou-Fiocruz, for the assistance on the statistical analysis, and to Vera de Paula Ribeiro, from Laboratório de Esquistossomose, Centro de Pesquisas René Rachou-Fiocruz, and Dr. John Kusel, from the Glasgow University, for English review.

\section{REFERENCES}

Bell DR 1963. A new method for counting Schistosoma mansoni eggs in faeces. Bull Wld Hlth Org. 29: 525-530.

Blagg W, Schloegel EL, Mansour NS, Khalaf GI 1955. A new concentration technique for the demonstration of protozoa and helminth eggs in faeces. Am J Trop Med Hyg 4: 23-28.

Corrêa-Oliveira R, Malaquias LCC, Falcão PL, Viana IRC, BahiaOliveira LMG, Silveira MAS, Fraga LAO, Prata A, Coffman RL, Lambertucci JR, Cunha-Melo JR, Martins-Filho AO, Wilson RA, Gazzinelli G 1998. Cytokines as determinants of resistance and pathology in human Schistosoma mansoni infection. Braz J Med Biol Res 31: 171-177.
Enk MJ, Lima AC, Massara CL, Coelho PMZ Schall VT 2008. A combined strategy to improve the control of Schistosomiasis mansoni in areas of low prevalence in Brazil. Am J Trop Med Hyg 78: 140-146.

Faust EC, Melency D 1924. Zinc sulphate centrifugal flotation method in diagnosis of parasitic infection. Am J Huf 3: 218-219.

Ferrari MLA, Coelho PMZ, Antunes CMF, Tavares CAP, Cunha AS 2003. Efficacy of oxamniquine and praziquantel in schistosomiasis mansoni treatment: a controlled trial. Bull World Health Organ 81: 190-196.

Gazzinelli A, Velasquez-Melendez G, Crawford SB, LoVerde, PT, Corrêa-Oliveira R, Kloos H 2006. Socioeconomic determinants of schistosomiasis in a poor rural area in Brazil. Acta Tropica 99: 260-271.

Hastings-Willis H 1921. A simple levitation method for the detection of hookworm ova. Med J Australia 29: 375-376.

Hoffman WA, Pons JA, Janer JL 1934. The sedimentation-concentration method in schistosomiasis mansoni. Puerto Rico J Publ Hlth Trop Med 9: 283-298.

Jurberg AD, Oliveira AA, Lenzi HL, Coelho PMZ 2008. A new miracidia hatching device for diagnosing schistosomiasis. Mem Inst Oswaldo Cruz 103: 112-114.

Kato K, Miura M 1954. Comparative examinations. Jap J Parasitol 3: 35 .

Katz N, Chaves A, Pellegrino J 1972. A simple device for quantitative stool thick-smear technique in schistosomiasis mansoni. Rev Inst Med Trop Sao Paulo 14: 397-400.

Katz N, Coelho PMZ, Pellegrino J 1970. Evaluation of Kato's method through the recovery of Schistosoma mansoni eggs added to human feces. J Parasitol 56: 5.

Lutz A 1919. Schistosomum mansoni e a schistosomatose segundo observações feitas no Brasil. Mem Inst Oswaldo Cruz 11: 121-155.

Ritchie LS 1948. An ether sedimentation technique for routine stool examinations. Bull US Army Med Dept 8: 326.

Ritchie LS, Berrios-Duran LA 1961. A simple procedure for recovering schistosome eggs in mass from tissues. J Parasitol 47: 363-365.

Rowan WB, Gram AL 1959. Quantitative recovery of helminth eggs from relatively large samples of feces and sewage. J Parasitol 45: 615-621.

Sapero JJ, Lawless DK, Strome CPA 1951. An improved iodinestaining technique for routine laboratory diagnosis of intestinal protozoa. Science 114: 550-551.

Stoll NR 1946. An efficient concentration method, Aex, for detecting helminthic ova in feces, modification of the Teleman Technic. Loughlin EH, Stoll NR. Am J Trop Med 26: 517-527.

Teixeira CF, Neuhauss E, Ben R, Romanzini J, Graeff-Teixeira C 2007. Detection of Schistosoma mansoni eggs in feces through their interaction with paramagnetic beads in a magnetic field. PLoS Negl Trop Dis 1: e73.

WHO - World Health Organization 1993. The control of schistosomiasis. Second report of the WHO Expert Committee. WHO Tech Rep Ser 830: 1-86.

WHO - World Health Organization 2006a. Report of the scientific working group meeting on schistosomiasis for the special programme for research and training in tropical diseases, WHO, Geneva, $123 \mathrm{pp}$.

WHO - World Health Organization 2006b. Report of the scientific working group meeting on schistosomiasis for the special programme for research and training in tropical diseases. In A Rabello, M Enk (eds.), Progress towards the detection of schistosomiasis, WHO, Geneva, p. 67-71. 